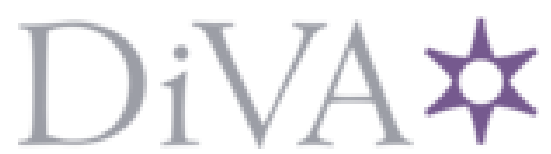

http://www.diva-portal.org

This is the published version of a paper published in IEEE transactions on dielectrics and electrical insulation.

Citation for the original published paper (version of record):

Ariza, D., Becerra, M., Hollertz, R., Wågberg, L., Pitois, C. (2017)

First Mode Negative Streamers along Mineral Oil-solid Interfaces.

IEEE transactions on dielectrics and electrical insulation, 24(4)

Access to the published version may require subscription.

N.B. When citing this work, cite the original published paper.

Permanent link to this version:

http://urn.kb.se/resolve?urn=urn:nbn:se:kth:diva-213422 


\title{
First Mode Negative Streamers along Mineral Oil-solid Interfaces
}

\author{
David Ariza1, Marley Becerra ${ }^{1,3}$, Rebecca Hollertz $^{2}$, Lars Wågberg ${ }^{2}$ \\ KTH Royal Institute of Technology. \\ ${ }^{1}$ Department of Electromagnetic Engineering, School of Electrical Engineering \\ ${ }^{2}$ Division of Fibre Technology, School of Chemical Science and Engineering \\ Stockholm, 10044, Sweden \\ and Claire Pitois \\ ${ }^{3}$ ABB AB Corporate Research \\ Forskargränd \\ Västerås, 72178, Sweden
}

\begin{abstract}
This document presents an experimental study on the propagation of first mode negative streamers along mineral oil-solid interfaces. Samples made of an oil impregnated kraft paper and a low-porosity paper made from cellulosic micro and nano fibrils, as well as different polymeric films (low density polyethylene (LDPE), polyethylene terephthalate (PET), polytetrafluoroethylene (PTFE) and polyvinylidene fluoride (PVDF)) are used as the solid. A comparison of the length, charge and velocity of streamers for all different mineral oil-solid interfaces is reported. It is shown that streamers propagate longer and faster along mineral oil-solid interfaces with low surface roughness, low porosity and higher electrical permittivity than mineral oil. Those streamers show a quasi-continuous injection of charge in the early stage of their propagation. This quasi-continuous charge injection consists of a sequence of small charge steps separated by few tens of nanoseconds in between. In comparison, the streamers that propagate along surfaces with similar permittivity to the mineral oil have lower injection of charge and higher stopping voltage conditions than streamers propagating free in the liquid without any solid barrier.
\end{abstract}

Index Terms - Streamers, creeping discharges, mineral oil, impregnated paper

\section{INTRODUCTION}

HIGH voltage components such as transformers, power capacitors and impregnated (oil-filled) cables include liquidsolid dielectric interfaces in their insulation systems. The recent development in nanotechnology and tailor-made solid materials has opened new possibilities to improve the dielectric performance of insulation systems. For instance, cellulose-based materials could be engineered to improve their dielectric performance. In order to design and implement new materials, it is required to understand the physical properties of the prebreakdown phenomena in liquids and along liquidsolid dielectric interfaces. Unfortunately, the physical mechanisms involved in the prebreakdown phenomena in liquids and along liquid-solid interfaces are not fully understood. As a consequence, there are still no predictive theory and models of breakdown in liquids comparable with those available for breakdown in gases [1]. This lack of understanding especially hinders the design of new insulating materials since it is still not clearly known either which or how the physical and chemical properties of a solid needs to be tailored in order to reach optimum dielectric performance.

The prebreakdown phenomena in liquids and along liquidsolid interfaces are generally referred to as streamers. Extensive research has been performed on streamers in liquids during the last decades [1], with some studies dedicated to their propagation along liquid-solid dielectric interfaces [214]. Particularly, technological advances in the performance of high speed cameras and optical techniques have been groundbreaking when it comes to the observations of streamers in liquids and along liquids-solid interfaces [1, 1517]. Schlieren and shadow photography combined with new electrical measurement techniques have allowed the measurement of the streamer parameters with high resolution. Observations of shape, length, velocity, charge, light emission, voltage gradient, among other streamer parameters, have also been reported based on these techniques [18-20].

Observations of streamers propagating along liquid-solid interfaces have shown that the streamers are influenced by the solid properties. The length, velocity, charge, current, light emitted and voltage gradient of streamers are some of the 
parameters affected by the presence of dielectric solid barriers [2-14, 21-28]. Strong indications of the influence of the permittivity and thickness of the solid on the mechanism of streamer propagation has been reported $[1,5]$. Nevertheless the effect of the several other (chemical and physical) properties of the solid on the propagation and stopping of streamers still remains unclear.

Streamers in dielectric liquids have been classified in different propagation modes depending on their propagation velocity $[1,29]$. The inception and propagation of first mode negative streamers are better understood than the first mode positive streamers. In negative polarity, the inception of a first mode streamers has been found to be caused by electron avalanches of nanosecond duration in the liquid phase [1, 30, 31]. The electron avalanche is responsible for the creation of the first gaseous cavity of the streamer. The propagation of negative streamers typically has been detected through a series of charge steps and current pulses due to partial discharges in the gaseous phase. Each charge step is correlated to one pulse of current and each pulse of current can result in the creation of a new gaseous cavity. Since the inception and propagation of the negative streamers in liquids is better understood, the study of first mode negative streamer propagation along liquid-solid interfaces can be used to identify possible properties of the solid that influence the propagation mechanism.

This work presents experimental results of first mode negative streamers propagating along mineral oil-solid interfaces. Six different materials are selected for the solid dielectric in order to compare the influence of the chemistry, permittivity and morphology of the solid on the streamer properties. Two different types of paper, one made of wood pulp and one from cellulosic micro and nanofibrils and four polymeric films are tested. Measurements of length, charge and velocity of streamers propagating along mineral oil-solid interfaces with different solid materials are reported and discussed.

\section{EXPERIMENTAL SETUP}

\subsection{CHAMBER AND ELECTRODE ARRANGEMENT}

Figure 1 introduces the experimental setup. It consists of a point-plane configuration assembled in a test chamber. The point electrode is a tungsten needle with tip radius $r_{p}$ of $2.9 \mu \mathrm{m}$. The gap distance $d$ is $5 \mathrm{~mm}$ and the plane electrode is $100 \mathrm{~mm}$ in diameter. The chamber is cylindrical with diameter of $200 \mathrm{~mm}$ and a height of $200 \mathrm{~mm}$. The needle tip is located vertical at the center of the chamber and aligned with the center of the lateral viewports. In order to protect the system in case of breakdown, the plane electrode is covered with an impregnated paper with a thickness of $100 \mu \mathrm{m}$.

\subsection{SAMPLE ASSEMBLING}

The solid is inclined 60 degrees with respect to the plane electrode and in contact with the needle tip as shown in Figure 2a. The solid is installed on a holder mounted on two mechanical stages with micrometrical resolution. The micrometrical stage moves the solid surface towards the needle tip with a resolution of $0.41 \mu \mathrm{m} /$ degree (micrometrical

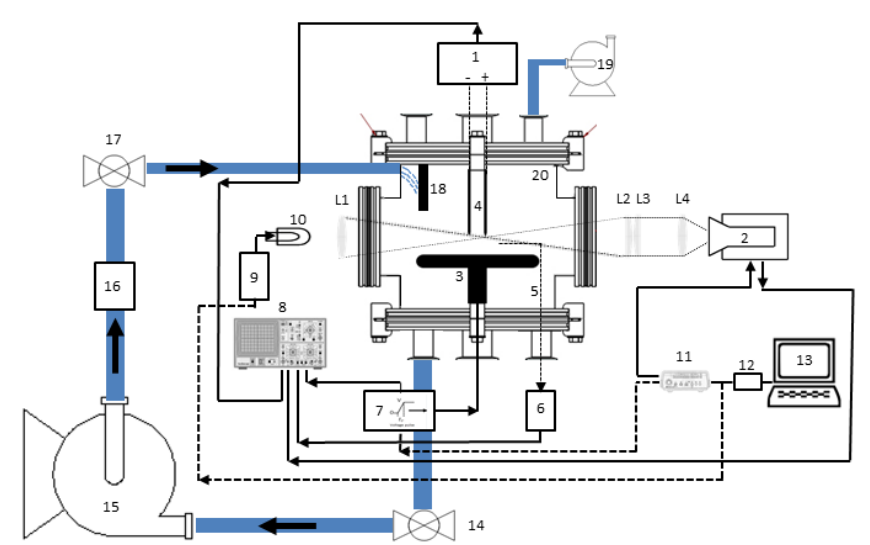

Figure 1. Schematic of the experimental setup. (1) Charge measuring system, (2) high speed camera, (3) plane electrode, (4) probe-plane and point-plane configuration, (5) fiber optic, (6) photomultiplier, (7) high voltage pulse source, (8) oscilloscope, (9) xenon lamp power source, (10) xenon lamp, (11) signal generator, (12) USB data acquisition device, (13) computer, (14) and (17) valves, (15) magnetic pump, (16) particles filter, (18) heating rod, (19) vacuum pump, (20) chamber.

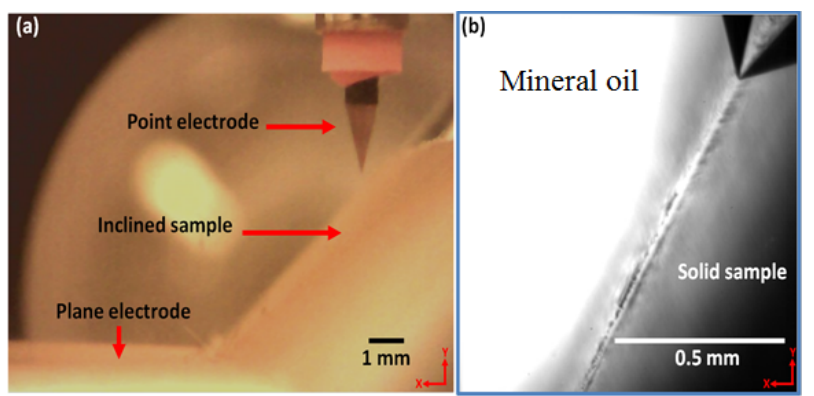

Figure 2. a) Point-plane arrangement with the solid surface. b) Shadowgraph (see 2.5 for details), with highest magnification, showing the solid surface in contact with the needle tip

screw control), until they are in contact. Figure $2 \mathrm{~b}$ shows a shadowgraph of the needle tip in contact with the solid surface (at the maximum magnification and resolution of the optical system).

\subsection{HIGH VOLTAGE PULSE SOURCE}

The high voltage pulse source is based on a $10 \mathrm{~V}-30 \mathrm{kV}$ DCDC voltage converter connected in parallel with $15 \mathrm{nF}$ capacitor bank. Square voltage pulses are generated by using an electronic push-pull switch, configured to generate pulses of positive polarity. The high voltage pulses are transmitted to the plane electrode with a $1 \mathrm{~m}$ long coaxial cable in order to avoid voltage reflections. The rise time $t_{r}$ and the fall time $t_{f}$ of the pulse is $35 \mathrm{~ns}$.

\subsection{STREAMER CHARGE MEASURING SYSTEM}

A large displacement current circulates in the arrangement during the rise and fall of each voltage pulse, charging the point electrode (point charge). If a streamer is generated, the total circulating charge is the summation of the charge injected by the streamer and the charge induced in the point electrode. In order to measure the charge of the streamer with high detection sensitivity, the point charge has to be rejected from the streamer charge measurement system. For this, a second 
non-sharp electrode referred to as probe electrode is introduced in the configuration. The probe electrode has similar geometry to the point electrode but with a radius tip of $500 \mu \mathrm{m}$ as shown in Figure 3. The probe and the point electrodes have a cylindrical shield around them in order to reduce the stray capacitance. Figure 4 shows a schematic of the circuit of the used differential amplifier system. $\mathrm{C}_{\text {probe }}$ and $\mathrm{C}_{\text {point }}$ represent the stray capacitance of the probe electrode and the point electrode respectively. The stray capacitances are calculated from the direct measurement of the point and the probe charge as $5.7 \times 10^{-14} \mathrm{~F}$ and $7.4 \times 10^{-14} \mathrm{~F}$ respectively. The streamer charge measuring system is based on two buffer amplifiers and an operational amplifier to subtract their signals. The input impedance of the charge measuring system is $10^{12} \Omega$. The resistors, $\mathrm{R} 1=\mathrm{R} 2=$ $68 \mathrm{M} \Omega$, stabilize the buffer amplifiers and avoid their saturation. The streamer charge measuring system is connected to the point and the probe electrodes with semirigid coaxial cables with lengths of $200 \mathrm{~mm}$. The capacitance of the semi-rigid coaxial cables including a SMA connector is $65 \mathrm{pF}$ at the point electrode line and $71 \mathrm{pF}$ at the probe electrode line. The capacitance of both $\mathrm{C} 1$ and C2 is $200 \mathrm{pF}$. The equivalent capacitance of the point electrode line is $265 \mathrm{pF}$ and defines a long time constant of integration $\tau=$ $18 \mathrm{~ms}$. The equivalent capacitance of the probe electrode line is $271 \mathrm{pF}$. The streamer measurement system compensates the differences in the parameters of each probe by controlling the gain, $\mathrm{G}$, of the probe electrode channel. The output of the differential amplifier is $\mathrm{V} 1-\mathrm{G} \cdot \mathrm{V} 2$, where $\mathrm{V} 1$ is the voltage induced in the point electrode and V2 is the voltage induced in the probe electrode. The gain, $G$, is tuned until the differential amplifier has a zero output under voltages pulses without streamers. The sensitivity of the charge measurement system is $0.3 \mathrm{pC}$. The streamer charge measurement system is designed and built following recommendations and techniques reported in $[1,18,30,32]$.

\subsection{SHADOWGRAPHIC SYSTEM AND PHOTON DETECTION}

The light emitted by first mode streamers has a very low intensity. Moreover, the content of aromatic compounds in mineral oil increases the transmission losses of any emitted light. For these reasons, the first and second mode streamers are generally observed using shadowgraphy and Schlieren techniques (e.g. [1, 2, 13, 19, 30, 33-43]). In this document, a shadow photographic arrangement is used. It consists of a light beam from a xenon lamp illuminating the point electrode tip such that a shadowgraph is recorded with a high speed camera. Lenses are used to magnify the image $20 \mathrm{X}$ and to project it on the camera sensor. The detected image size is $1000 \times 1000 \mu^{2}$ with a maximum resolution of $1 \mu \mathrm{m} /$ pixel.

An optical fiber with a diameter of $2 \mathrm{~mm}$ is used to transmit photons emitted by the streamer towards a photomultiplier tube. The fiber optic faces the tip of the point electrode, 15 $\mathrm{mm}$ away from it. The photomultiplier has a spectral sensitivity ranging between 200 and $700 \mathrm{~nm}(10 \%$ of sensitivity at $650 \mathrm{~nm})$. In order to minimize the interference

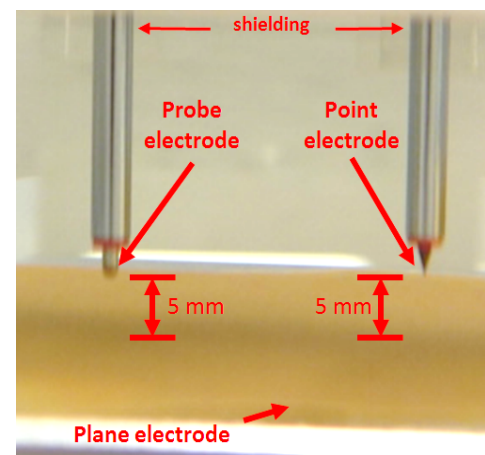

Figure 3. Point-plane and probe-plane arrangement immersed in mineral oil. Gap distance $\mathrm{d}=5 \mathrm{~mm}$.

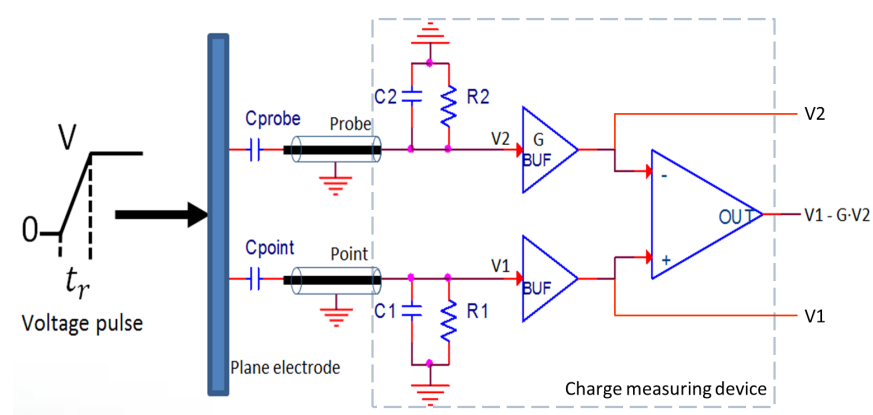

Figure 4. Schematic circuit of the charge measurement system. $\mathbf{R} 1=\mathbf{R} 2=$ $68 \mathrm{M} \Omega, \mathbf{C 1}=\mathbf{C 2}=\mathbf{2 0 0} \mathbf{p F}$. $\mathrm{C}_{\text {probe }}$ and $\mathrm{C}_{\text {point }}$ are the capacitance between the probe and the plane and the point and the plane.

of the shadowgraphic system with the photon detection, the line of sight of the optical fiber is set to 120 degrees from the xenon lamp light beam. In addition, a longpass filter with cuton wavelength of $650 \mathrm{~nm}$ is installed in front of the xenon lamp. The filtered light beam from the xenon lamp is scattered in the liquid introducing a noise level lower than $5 \%$ of the total voltage range of the photomultiplier. The photomultiplier is installed inside of a dark shielding box to minimize electromagnetic interference.

\subsection{FILTERING AND DEGASSING}

Before starting a test, the chamber is half filled with mineral oil Nitro 10X supplied by Nynas AB, Stockholm, Sweden (enough to cover the point-plane arrangement). A magnetic pump circulates the mineral oil during the filtering and degassing process in a closed loop indicated for the arrows in Figure 1. The mineral oil is pumped through a filter with pore size of $2 \mu \mathrm{m}$. In order to improve the degassing of the oil, it is poured on the surface of a $60^{\circ} \mathrm{C}$ heating rod when it circulates back into the chamber. Furthermore, a vacuum pump is used to reduce the pressure of the test chamber to 5 mbar during the circulation of the oil. When the filtering and degassing process is finished after $24 \mathrm{~h}$, the oil circulation is stopped. Dry air ( $80 \%$ nitrogen and $20 \%$ oxygen) is inserted in the test chamber until atmospheric pressure is reached. The oil cools down to room temperature before the electrical tests. 


\section{SOLID SAMPLES}

\subsection{DESCRIPTION}

Polymeric films of low density polyethylene (LDPE ET311201), polyethylene terephthalate (PET ES301400), polytetrafluoroethylene (PTFE FP301300), and polyvinylidene fluoride (PVDF FV301300), supplied by Goodfellow Cambridge Ltd, Huntingdon, England, are used. The reference paper sample used is produced from kraft pulp while a low-porosity paper is made from cellulose micro and nanofibrils. The kraft pulp is kindly supplied by Aspa Bruk, Askersund, Sweden, Munksjö AB, containing around 75 weight $\%$ cellulose (it also contains approximately 3 weight $\%$ lignin and 15 weight $\%$ hemicellulose) determined by carbohydrate analysis [44]. The cellulose nano and microfibrils, here after denoted kraft fibrils, produced from the kraft pulp are prepared by beating the pulp for a total of 6000 revolutions in a PFI-mill and thereafter fluidizing at a pressure of $1600 \mathrm{bar}$ in a Microfluidizer M-110eh (Microfluidics Inc, USA). Defibrillation is achieved through three passes through a $400 \mu \mathrm{m}$ and a $200 \mu \mathrm{m}$ chamber connected in series followed by three passes through a $200 \mu \mathrm{m}$ and a $100 \mu \mathrm{m}$ chamber connected in series. Papers from kraft pulp (beaten for a total of 4000 revolutions in a PFI-mill) and kraft fibrils are produced using a Rapid Köthen sheet former (PTI, Pettenbach, Austria) according to procedures previously described in [44, 45]. All the investigated samples have a thickness of $100 \mu \mathrm{m}$.

The repeating units (elementary structural unit of a polymeric chain) of the polymers and the cellulose are shown in Figure 5. PTFE and PVDF contain four and two fluorine atoms per repeating unit respectively. Due to the asymmetric structure of PVDF, it has a high dipole moment compared to the symmetric PTFE. Thus, PVDF has a significantly higher relative permittivity compared to PTFE (8.4 compared to 2.02.1). PET has a relative permittivity around 3.0 , similar to the permittivity of the oil-impregnated paper. Both LDPE and PTFE have a relative permittivity about 2.0 , slightly lower that of mineral oil (2.2).

The relative permittivity of the impregnated kraft fibril paper (4.5) is higher than for the impregnated kraft paper (3.2) due to its lower porosity (i.e. lower fraction of mineral oil in the impregnated system). The papers are made of an impregnatable network of wood fibres or fibrils in order to have both a low density, high porosity, and a high density, low porosity, paper respectively. In comparison, for a polymeric film a high density normally refers to more closely packed polymeric chains and less free-volume and chain mobility.

\subsection{CHARACTERIZATION}

The surface morphology of the solid samples is investigated with scanning electron microscopy (SEM, Hitachi S- 4800) and atomic force microscopy (AFM, Nanoscope IIIa AFM, Bruker AXS). The average roughness of the AFM measurements is defined as the root mean square of the values measured over three different areas $15 \times 15 \mu \mathrm{m}$ in size. Sample properties (relative permittivity, surface roughness and

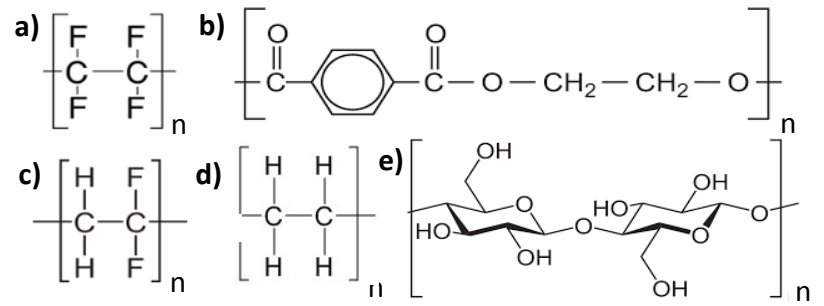

Figure 5. The repeating units of a) polytetrafluoroethylene (PTFE), b) polyethylene terephthalate (PET), c) polyvinylidene fluoride (PVDF), d) polyethylene (PE) and e) cellulose (the dominating wood polymer, in content, in wood pulp).
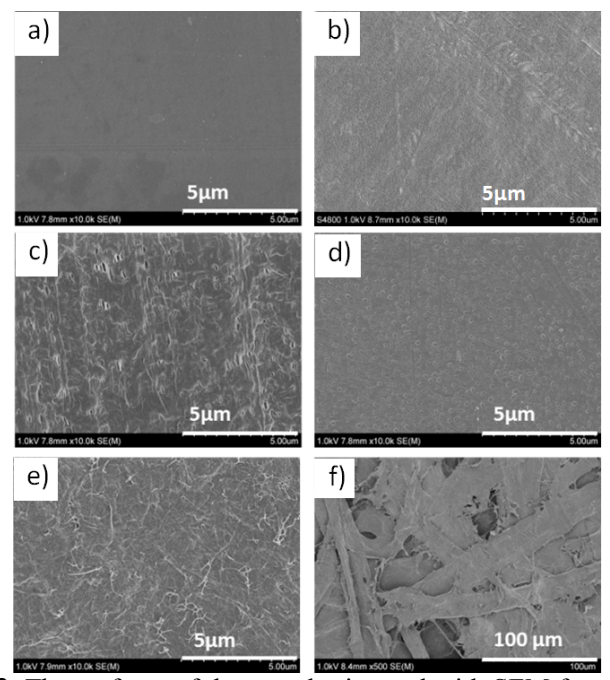

Figure 6. The surfaces of the samples imaged with SEM for a) PET, b) LDPE, c) PTFE, d) PVDF, e) kraft fibril films and f) paper made from a kraft pulp. Note that the magnification is decreased from x10 000 to $\mathrm{x} 500$ for the kraft paper in order to be able to observe its coarser structure.

Table 1. Sample Properties

\begin{tabular}{c|c|c|c}
\hline Material & $\begin{array}{c}\text { Relative } \\
\text { permittivity } \\
\epsilon_{r}\end{array}$ & $\begin{array}{c}\text { Average surface } \\
\text { roughness }(\mathrm{nm})\end{array}$ & $\begin{array}{c}\text { Density } \\
\left(\mathrm{g} / \mathrm{cm}^{3}\right)\end{array}$ \\
\hline LDPE & $2.2^{1}$ & 30 & $1.30^{1}$ \\
PET & $3.0^{1}$ & 3 & $1.92^{1}$ \\
PTFE & $2.0-2.1^{1}$ & 130 & $2.20^{1}$ \\
PVDF & $8.4^{1}$ & 360 & $1.76^{1}$ \\
Kraft fibril paper & $4.5^{2}$ & 350 & 1.30 \\
Kraft paper & $3.2^{2}$ & 2000 & 0.80 \\
Oil & $2.2^{2}$ & - & $0.87^{1}$ \\
\hline
\end{tabular}

${ }^{1}$ From supplier

${ }^{2}$ Measured by dielectric spectroscopy (procedure described in [44])

density) are summarized in Table 1. The thicknesses used for calculating the densities of the papers are averages from 5 measurements with a disc micrometer (Absolute Digimatic Quick 227, Mitutoyo) of a stack of 3 test pieces (derived from ISO 534:2005). Images of the different surface morphologies, of the different samples, obtained with SEM analysis are shown in Figure 6.

\subsection{PREPARATION AND IMPREGNATION}

The solid samples are cut into long narrow pieces of $8 \times 100 \mathrm{~mm}$. The samples are heated in a vacuum oven at 105 
${ }^{\circ} \mathrm{C}$ under low pressure (5 mbar) to remove all moisture which is essential specially for the cellulose-rich samples. The drying procedure is done at $70{ }^{\circ} \mathrm{C}$ for the LDPE sample due to its low melting point. Filtered transformer oil is then inserted into the closed oven through a feed-thru after the temperature in the oven is reduced to $60^{\circ} \mathrm{C}$. The oil is degassed in a second glass container inside the oven. After $24 \mathrm{~h}$ of degassing, the oil is poured on the solid sample for impregnation.

\section{TESTING PROCEDURE}

After the solid sample is assembled, electrical tests are performed. The electrical tests consist of a series of square high voltage pulses applied to the point-plane arrangement. The high voltage pulses have $35 \mathrm{~ns}$ of rise time and duration of $40 \mu \mathrm{s}$. The voltage range applied varies between $11.5 \mathrm{kV}$ and $22 \mathrm{kV}$ with steps of $0.6 \mathrm{kV}$ approximately. Four series of 10 measurements each are performed for each voltage level. The waiting time between each shot is $60 \mathrm{~s}$. In order to detect possible conditioning of the samples, the point electrode or the oil during tests, the experiment is randomized by selecting the peak voltage for each series randomly. Analysis of the results for the series at all tested voltage levels shows that no significant alteration of either the solid, the point electrode or the oil potentially influencing the experiment are produced during the entire test. This means that each voltage pulse applied in the experiment can be considered stochastically independent.

\section{CHARACTERIZATION OF FIRST MODE NEGATIVE STREAMER}

\subsection{STREAMER CHARGE AND LIGHT INTENSITY RECORDINGS}

The propagation of a first mode negative streamers consists of the successive generation of gaseous cavities initiated at the tip of the point electrode [37]. The gaseous phase is a consequence of the energy dissipated by an electron avalanche of nanosecond duration in the liquid phase [30]. Once the gaseous phase is formed, a series of electrical discharges takes place inside of the cavity. These electrical discharges generate a series of current pulses correlated with the re-illuminations of the gaseous cavity. Each current pulse can result in the generation of a new cavity [1] and its corresponding charge step represents the injection of charge to the gaseous volume [37]. The number of charge steps and re-illuminations vary stochastically for a fixed voltage and also increases by increasing the applied voltage.

Figure 7a shows typical recordings for a first mode negative streamer propagating in mineral oil without any solid. As it can be seen, this streamer had three main pulses of light correlated with three charge steps. Since the applied voltage, $21.7 \mathrm{kV}$, is much higher than the inception voltage $(12.2 \mathrm{kV})$, it is not possible to distinguish from the charge recordings when the electron avalanche occurs and when the gaseous cavity is formed. It is only possible to observe that both processes occur during the first 300 nanoseconds when the first pulse of light and a well defined first charge step are detected. After the first charge step injection, this streamer
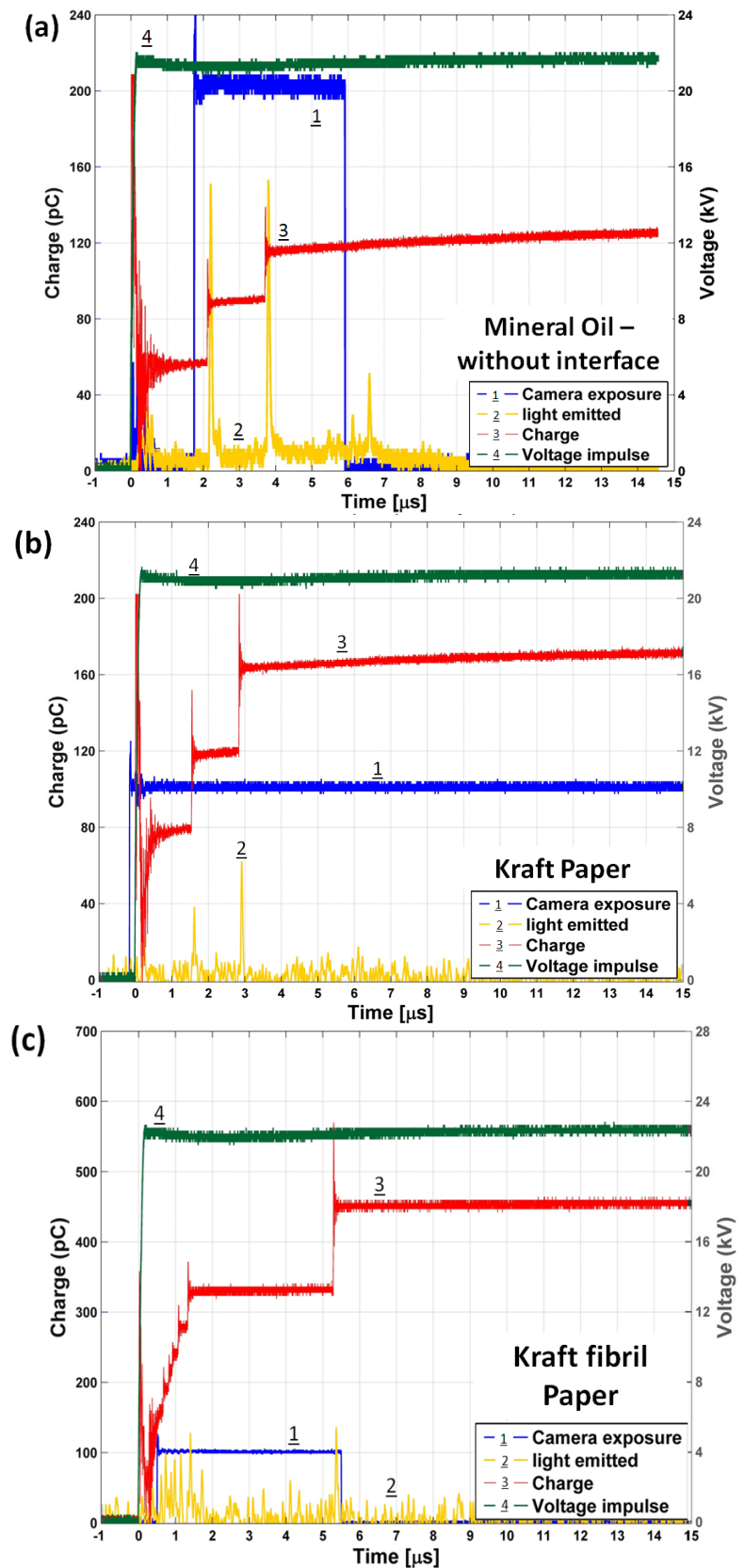

Figure 7. Typical charge and light recordings for the streamers propagating in mineral oil and along mineral oil-solid interfaces: (a) without interface, with (b) kraft paper and (c) kraft fibril paper.

presented two re-illuminations correlated with two charge steps. They are probably responsible for the propagation of the streamer by the generation of two additional gaseous cavities.

Typical charge and light recordings for a streamer propagating along the mineral oil-kraft paper interface are shown in Figure $7 \mathrm{~b}$. The charge and light signals recorded with the kraft paper have a similar appearance as in the case without solid in Figure 7a. Both cases presented a clear charge step in the early stage of the streamer propagation. In contrast to the cases without solid and with the kraft paper, the welldefined first charge step it is not observed anymore in the case of the kraft fibril paper, as shown in Figure 7c. The first injection of charge in this case consists of a sequence of small 

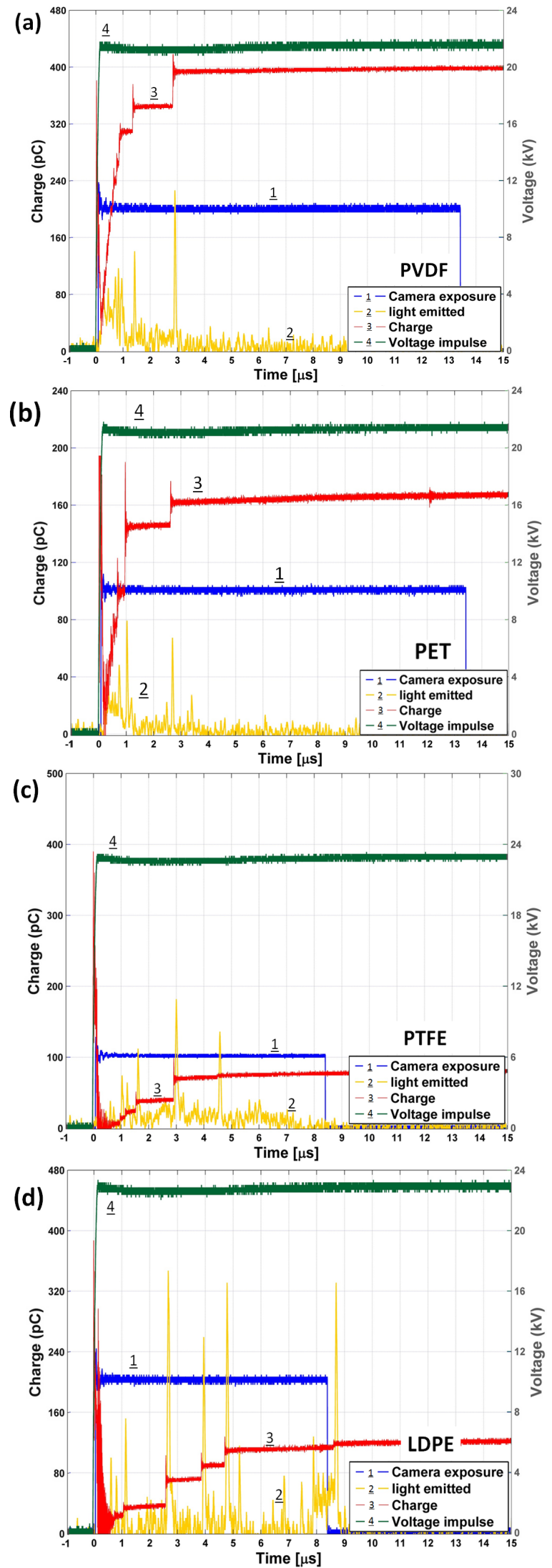

Figure 8. Typical charge and light recordings for the streamers propagating along mineral oil-solid interfaces with: (a) PVDF, (b) PTFE and (c) LDPE.

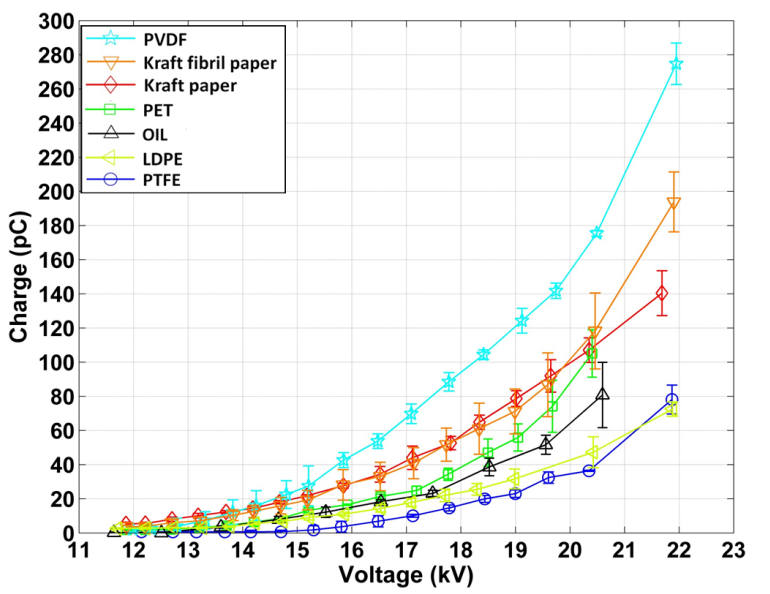

Figure 9. Average injection of charge of first mode negative streamers propagating in mineral oil and along mineral oil-solid interfaces. Solid interface inclined 60 degrees to the plane electrode. $d=5 \mathrm{~mm}, t_{r}=35 \mathrm{~ns}$, $r_{p}=2.9 \mu \mathrm{m}$.

charge steps separated by few tens of nanoseconds in between. This sequence defines a quasi-continuous charge injection during the early stage of the development of streamers propagating along the kraft fibril paper. Since the kraft fibril paper has the same chemical composition as the kraft paper, the differences in the recordings between these two interfaces are attributable to the structural differences between these two materials (Table I).

In order to compare the influence of the permittivity and the surface roughness of the solid on the streamer propagation, charge and light recordings of polymeric materials are also measured as shown in Figure 8. The measurements with PVDF, which has the highest relative permittivity $\left(\epsilon_{r}=8.4\right)$ of all the materials tested, also show a quasi-continuous injection of charge during the early stage of the streamer propagation (Figure 8a). A quasi-continuous light signal during this early stage is clearly observed in this case. It is followed by a series of few charge steps correlated with light pulses. Similar features are also observed for PET in Figure 8b, which has a slightly lower relative permittivity $\left(\epsilon_{r}=3\right)$ than the impregnated kraft paper $\left(\epsilon_{r}=3.2\right)$. Interestingly, the sequences of small charge steps of the quasi-continuous injection of charge with PVDF and PET have shorter separation time than with kraft fibril paper. These similarities during the early propagation of streamers along the kraft fibril paper and the PVDF and PET films appear to occur for solids with low surface roughness and higher permittivity than mineral oil. Nevertheless, it should be noted that PVDF (highest permittivity) does not show significant difference on the quasi-continuous injection of charge compared to PET. This means that higher permittivity than mineral oil is one of the conditions of the solid to induce a quasi-continuous injection of charge but is not the most influent.

In order to assess the conditions of streamer propagation when the permittivity of the solids and the mineral oil are matched, PTFE and LDPE films are also tested. The results in Figures $8 \mathrm{c}$ and $8 \mathrm{~d}$ show that the permittivity matching of the liquid-solid interface leads to a low injection of charge during the early propagation of streamers. 

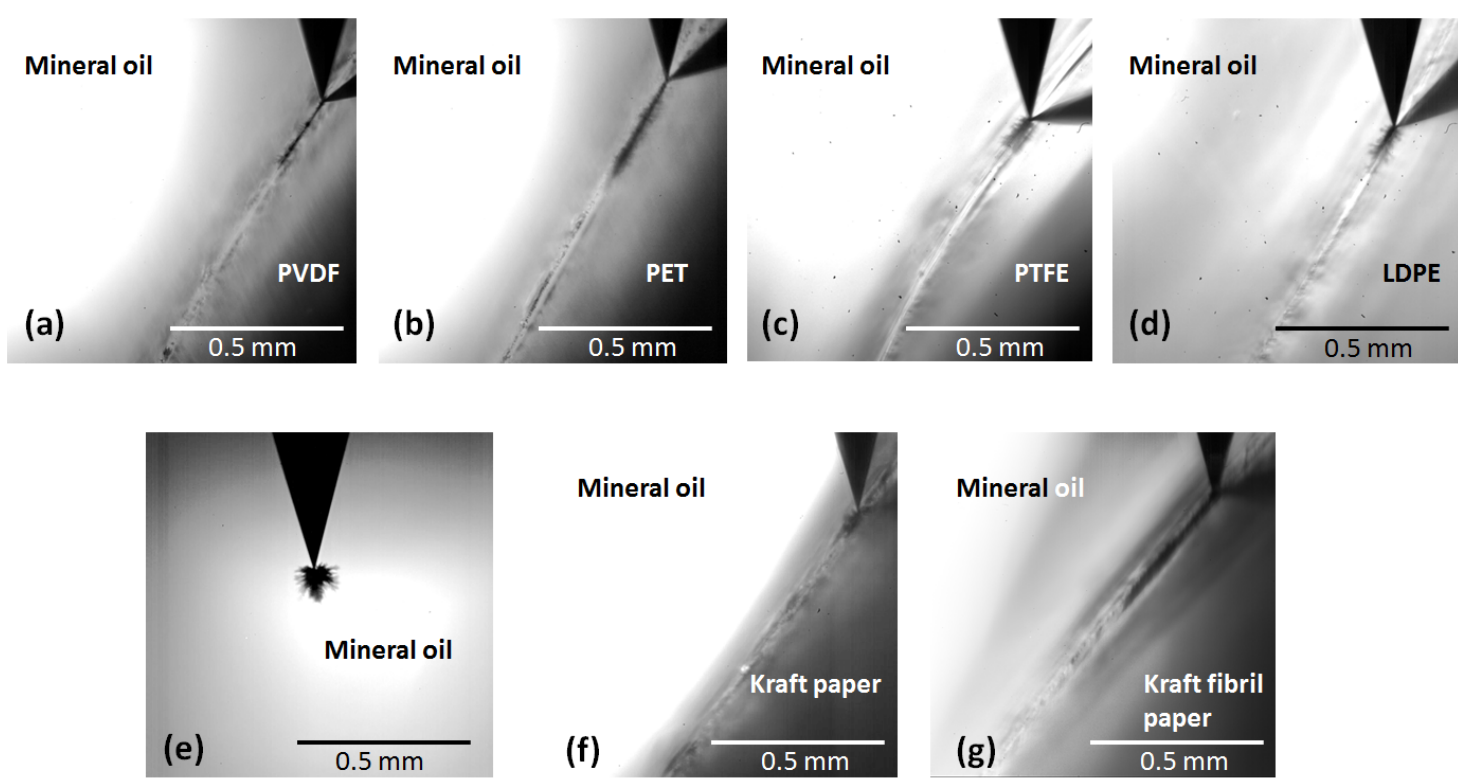

Figure 10. Shadowgraph of first mode negative streamers propagating in mineral oil and along different combinations of mineral oil-solid interfaces with: (a) PVDF, (b) PET, (c) PTFE, (d) LDPE, (e) No solid barrier, (f) kraft paper and (g) kraft fibril paper. The solid surface is inclined 60 degrees to the plane electrode. $d=5 \mathrm{~mm}, t_{r}=35 \mathrm{~ns}, r_{p}=2.9 \mu \mathrm{m}$.

Due to the low amplitude of the injected charge during this early stage, it is difficult to assess whether a first step or a quasi-continuous injection of charge takes place with the PTFE and LDPE cases.

\subsection{AVERAGE INJECTED STREAMER CHARGE}

The average injected streamer charge as a function of the applied voltage is shown in Figure 9. The average streamer charge shows an exponential correlation with the applied voltage. Notable is that the average injected charge increases by increasing the permittivity of the solid. Thus, the kraft fibril paper, kraft paper, PVDF and PET cases have a larger injection of charge than in the case without solid. The charge injection is lower for the PTFE and LDPE cases. It is important to note that even though the kraft paper and kraft fibril paper cases had very different shapes on their charge recordings they have very similar average injection of charge in Figure 9. These results show that a quasi-continuous injection of charge during the early stage of the streamer propagation could lead to an increment in the streamer charge injected, but it is not the only parameter responsible of this increment.

\subsection{STREAMER SHADOWGRAPHS}

Typical shadowgraphs of first mode negative streamers propagating in mineral oil and along the studied mineral oilsolid interfaces are shown in Figure 10. The shadowgraphs show the shape of the streamers at their stopping length. They correspond to the same events reported earlier, with the exposure time of the camera shown as trace 1 in Figures 7 and 8. Figure $10 \mathrm{e}$ shows a first mode negative streamer propagating in mineral oil without solid barrier. It consists of an ionized gaseous cavity of irregular shape. For the cases with a solid dielectric, the successive generation of gaseous cavities develops into a single main cavity of filamentary appearance propagating along the interface. Since the first mode negative streamers consist of an ionized gaseous cavity with pressure in quasi-equilibrium with the surrounding liquid [1], possible expansion of the cavity in the perpendicular plane of the shadowgraphs has to be considered.

\subsection{STREAMER LENGTH}

Figure 11 shows the streamer stopping length measured from the shadowgraphs as a function of the applied voltage. A linear correlation between the streamer length and the voltage applied is found. Similar correlation have been reported by other authors for faster streamers [4, 5, 8]. This linear correlation indicates a constant potential gradient along the channel of the streamer $[1,3,4,8,21]$. The mean potential gradient (E), i.e. $(\mathrm{kV} / \mathrm{mm})$, can be calculated from the data in Figure 11 and is reported in the legends for each material. Interestingly, the mean potential gradient is similar for the PVDF, PET, PTFE and kraft fibril paper cases despite of their large differences in permittivity. The cases with the LDPE film, kraft paper and without the interface have the highest potential gradient.

The streamer propagation is longer with the solids with higher permittivity and low surface roughness. It is, however, important to note that with PVDF the streamer did not show any significant differences in length compared to with PET or the kraft fibril paper which have significantly lower permittivity.

It is known that the streamer propagation stops when the electrical potential of the channel tip reaches a specific value [3], referred to as the stopping voltage. It is possible to estimate the stopping voltage from the intersection of the curves in Figure 11 with the voltage axis. Thus, the stopping voltages are found to be approximately $14.7 \mathrm{kV}$ for PTFE, $13.3 \mathrm{kV}$ for LDPE and $12.2 \mathrm{kV}$ with all the other investigated materials. It is interesting to observe that the stopping voltage condition of the channel tip does not significantly change even when the streamer propagates along surfaces with different permittivity. This reflects the importance of the liquid phase on the stopping condition of streamers propagating along the 


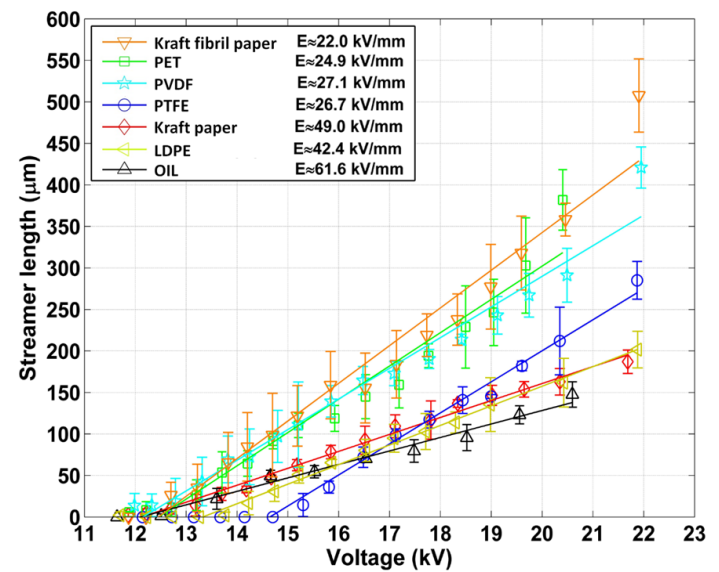

Figure 11. Stopping length of first mode negative streamers propagating in mineral oil and along mineral oil-solid interfaces. Solid surface inclined 60 degrees to the plane electrode. $d=5 \mathrm{~mm}, t_{r}=35 \mathrm{~ns}, r_{p}$ $=2.9 \mu \mathrm{m}$.

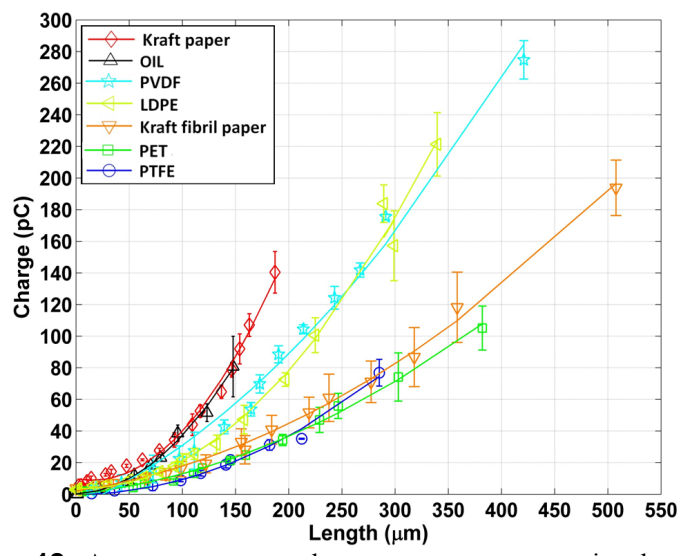

Figure 12. Average streamer charge vs streamer stopping length for first mode negative streamers propagating in mineral oil and along mineral oil-solid interfaces.

liquid solid interface. Nevertheless it is not possible to conclude that the stopping voltage condition is inherently independent of the solid material due to the differences seen for the cases with PTFE and LDPE.

Figure 12 shows the streamer charge as a function of the stopping length. A nonlinear dependency is found for streamers that propagate a short distance from the electrode tip up to $130 \mu \mathrm{m}$ with PVDF, PET and kraft fibril paper and 75 $\mu \mathrm{m}$ with LDPE, PTFE, kraft paper and the case without interface. For the streamers that propagate longer distances, the correlation between the charge and the length tends to be linear. This linear dependency has been reported by other authors in $[19,21]$. It is interesting that the streamer charge per unit length recorded for the different cases are clustered into three different groups. The highest charge per unit length of the streamer is found for the cases without the interface and with the kraft paper. Streamers propagating along polymeric surfaces of PTFE, PET and the kraft fibril paper have the lowest values of charge per unit of length. The results for PVDF and LDPE are grouped together in between these two groups.

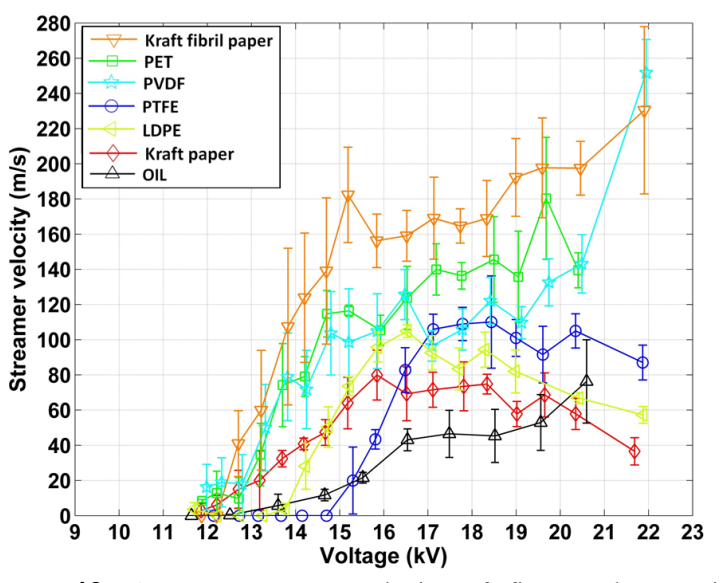

Figure 13. Average streamer velocity of first mode negative streamers propagating in mineral oil and along mineral oil-solid interfaces.

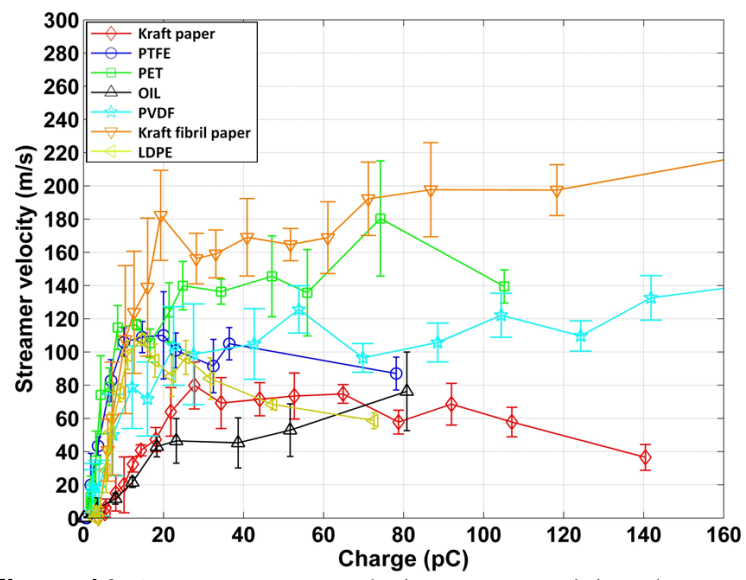

Figure 14. Average streamer velocity vs average injected streamer charge of first mode negative streamers propagating in mineral oil and along mineral oil-solid interfaces.

\subsection{STREAMER VELOCITY}

Figure 13 shows the average streamer velocity as a function of the applied voltage for all the cases. The reported average velocity is roughly estimated from the maximum streamer length measured from the shadowgraphs and the time when the last streamer charge injection is detected. The velocities reported range between $10 \mathrm{~m} / \mathrm{s}$ and $280 \mathrm{~m} / \mathrm{s}$. Negative streamers propagating under such velocities are generally classified in the first mode of propagation [1,29]. The average velocity curves as a function of the applied voltage have two major regions. A first region where the velocity increases linearly with the voltage and a second part with a rather constant velocity (this assumption is valid only for the range of voltage applied). Under higher voltages, an increment in the velocity and changes of mode propagation are expected. It is possible to estimate from Figures 11 and 13 that the transition between the linear and the constant regions of the velocity curves occurs when the streamer length is about $130 \mu \mathrm{m}$ with PVDF, PET, and kraft fibril paper and about $75 \mu \mathrm{m}$ with LDPE, PTFE, kraft paper and the case without interface. The average streamer velocity as a function of the average streamer charge injected is shown in Figure 14. All the curves 
present a linear correlation between the velocity and the average charge injection of streamers for charges up to about 20 pC. After this, the streamer velocity tends to be roughly constant and independent from the charge injected in most of the cases. Only the LDPE case presents an appreciable decrement in velocity as the injected charge increases.

\section{DISCUSSION}

The propagation of the first mode negative streamer along liquid-solid interfaces, as here reported, is influenced by the solid in different ways. First, the solid can cause electric-field intensification close to the point electrode or the streamer head (front of the gaseous cavity) [11]. This field intensification is induced by the permittivity mismatch between the solid and mineral oil [46]. Second, the interface also represents an electrical boundary where charges can get trapped, induced and accumulate, modifying the existing electric field and limiting the space charge [2]. Third, the solid surface acts as a geometrical barrier, limiting the volume where the streamer can propagate in front of the point electrode tip $[2,11]$. Forth, irregularities on the solid surface may influence the propagation of streamers at a microscopic level.

The kraft fibril paper, PVDF and PET solids have the highest permittivity mismatch with mineral oil. The results presented in section 5.1 showed that these solids affected the streamer charge injection mechanism. The charge recordings in these cases have a quasi-continuous charge injection in the early stage of the streamer development. This quasicontinuous charge injection is formed by a sequence of small charge steps separated by few tens of nanoseconds in between. The frequent electrical discharges of the quasi-continuous charge injection generate current pulses that can create new gaseous cavities or contribute to the expansion of the ones already existing [37, 47]. This quasi-continuous charge injection contributes to the increment of the average length and velocity of the streamer. As shown in Figures 11 and 13, the kraft fibril paper, PVDF and PET cases have the larger average length and velocity of the streamer. Moreover, the increment in the streamer velocity is a clear indication that the streamer propagation mechanism has been affected by the solid.

The permittivity mismatch between the solid interface and the mineral oil affects the electric field conditions for the gaseous cavity formation. First, the observed quasi-continuous charge injection is a consequence of the enhancement of the electric field in front of the gaseous cavity. Second, the observed quasi-continuous charge injection leads to an increment in the conductivity of the gaseous cavity. This is consistent with the reduction of the potential gradient of the streamer for the kraft fibril paper, PVDF and PET cases in Figure 11. Based on these results the quasi-continuous charge injection can be suggested to be a consequence of the permittivity mismatch of the liquid-solid interface and the enhancement of the streamer length and velocity can be correlated this difference in permittivity. Thus, permittivity mismatch is one of the parameters that influences the propagation of first mode negative streamers, as previously suggested for faster (higher mode) streamers [5].

On the other hand, the current flowing through the streamer is related to the displacement current through the solid and the liquid phase to the plane electrode. Thus, the presence of a solid with high permittivity also represents an increment of the capacitive coupling of the streamer to the opposite electrode [1]. Since the current of the streamer increases with the increment of the capacitive coupling [48], the streamer charge injection also increases. This is consistent with Figure 9 where it is shown that the average streamer charge increases with the permittivity of the solid. Figure 11 shows that for streamers propagating on the kraft fibril paper, PVDF and PET the potential gradient is smaller than for streamers propagating in mineral oil without solid. The reduction of the potential gradient is consistent with the increment of the capacitive coupling of the streamer $[1,3]$ and the conductivity of the streamer. Observations done for faster and longer streamers propagating along different liquid-solid interfaces in [5] and [49] also suggest that capacitive coupling of the streamer causes an increment of the streamer length.

However, a high permittivity is not the only common parameter for the kraft fibril paper, PVDF and PET; they also have a very low surface roughness. The results presented in Figures 11 and 13 do correlate the increment of the streamer length and velocity with the solids with high permittivity and low surface roughness. When a streamer propagates along surfaces with low roughness, the physical obstruction produced by surface irregularities can be neglected. Also, possible enhancement of the field due to localized protuberances on the surface [46] can be neglected.

A clear contrast in the surface roughness can be observed between the kraft paper and the other solid materials in Figure 6. Kraft paper is a fibrous impregnatable network (Figure 6e) where the transition between the bulk oil and the bulk impregnated paper composite is not well-defined [50]. Thus, the propagation of individual streamer filaments can be influenced by dimples and individual fibers (shown in Figure $6 \mathrm{e}$ ) on the surface of the kraft paper. Notice that the surface roughness of kraft paper reported in Table 1 is comparable with the streamer length reported in Figure 11. Thus, the physical obstruction of gaseous cavities due to surface irregularities on the kraft paper could be responsible for the short propagation of streamers in this case compared to the cases with the kraft fibril paper, PVDF and PET. Furthermore, this obstruction to the longitudinal propagation of a streamer can result in the creation of new cavities and branches in the transverse direction as in Figure 10f. The expansion and creation of new cavities leads to the increase of the average injected streamer charge. This affirmation can be supported by the comparison of the average streamer charge reported in Figure 9 for the kraft paper and the kraft fibril paper. Both cases have similar average charge injection even though streamers, propagating along the kraft paper, did not propagate as far as those propagating along the kraft fibril paper, PVDF and PET cases. Nevertheless, more investigations are needed to clarify the correlation of the large injection of charge and the short streamer propagation in the case with kraft paper as solid. 
On the other hand, the short propagation of the streamers along the kraft paper surface is also correlated to the absence of a quasi-continuous charge injection during their early stage. This means that the electric field condition for the formation of the gaseous cavity is not affected by the field intensification caused by the surface irregularities on the kraft paper surface. The gaseous cavity formation is mainly affected by the increment of its capacitive coupling to the opposite electrode due to the higher permittivity of the kraft paper. The absence of a quasi-continuous charge injection for the kraft paper case is also correlated to its high surface roughness. Kraft paper and kraft fibril paper basically have the same chemistry and both have higher permittivity than the mineral oil. Nevertheless, the kraft fibril paper has a surface roughness about seven times smaller than the kraft paper. The kraft fibril paper showed a clear quasi-continuous charge injection in the early stage of the streamer propagation while the kraft paper case showed a well-defined charge step injection (section 5.1). This comparison leads to the conclusion that the combination of low surface roughness and higher permittivity than the mineral oil are essential condition of the solid for the increment of the streamer length, velocity and the quasicontinuous charge injection of first mode negative streamers propagating along liquid-solid interfaces.

PTFE and LDPE have similar permittivity to the mineral oil. For this reason, the streamer capacitive coupling effect is reduced compared to the cases with the other solids. Additionally, the permittivity mismatch between the solid and the mineral oil is neglected such that no further enhancement of the field due to irregularities on the solid surface is expected [46]. All these effects lead to the lowest charge injection for the streamers propagating along the PTFE and LDPE surfaces as it is reported in Figure 9. Moreover, the presence of PTFE and LDPE limits geometrically the volume where streamers can propagate.

The potential gradient distribution is also affected by the geometrical restriction of the streamer volume. Thus, the streamer potential gradient, reported in Figure 11, is lower for the PTFE $(26.7 \mathrm{kV} / \mathrm{mm})$ and the LDPE $(42.4 \mathrm{kV} / \mathrm{mm})$ interfaces compared with the case without solid interface (49 $\mathrm{kV} / \mathrm{mm}$ ). Interestingly, the conductivity of streamers propagating along the PTFE surface is higher than for those propagating along the LDPE surface. Since the permittivity, capacitive coupling and geometrical restriction of the PTFE and LDPE interfaces are similar, the differences in the electrical conditions of the streamer in these two cases could mainly be explained by accumulation of surface charge on the solid surface $[11,51]$. The accumulation of charges on the solid surface produced by the interaction of the streamer and the interface could produce a change in the field in front of the streamer head. In addition, the geometrical restriction of the streamer volume forces to the redistribution of the streamer charge. This redistribution of the charges modifies the electric potential in front of the gaseous cavity (streamer head). The ability of the solid surface to accumulate charges also should influence the electric potential of the gaseous cavity. This result is consistent with the stopping voltage estimated in Figure 11 as $14.7 \mathrm{kV}$ for PTFE and $13.3 \mathrm{kV}$ for LDPE case.
The modifications of the field in front of the streamer head should also result in a modification of the streamer velocity because it affects the streamer propagation mechanism. This is consistent with the results in Figure 13 showing that streamers propagating along PTFE and LDPE surfaces are faster than streamers propagating free in the liquid volume without solid. The increment of the streamer velocity due to the presence of a solid with similar permittivity than the liquid has been also reported by other authors in [11].

On the other hand, it is surprising that the stopping voltage condition is the same for the interfaces with PVDF, PET, kraft fibril paper, kraft paper and without solid $(12.2 \mathrm{kV})$. For these cases the stopping voltage condition does not significantly change when the streamer propagates along surfaces with different permittivity and chemistry; the streamer stopping voltage condition appears to be mainly related to the liquid phase. Nevertheless the stopping voltage condition is not inherently independent of the solid material since the results with PTFE and LDPE show that low permittivity of the solid is a possible condition that increase the streamer stopping voltage.

Regarding the velocity of the negative streamers in the experiment, the results in Figure 13 show that they are classified in the first mode of propagation [1, 29]. The first mode negative streamers consist of an ionized gaseous cavity of irregular shape with pressure in quasi-equilibrium with the surrounding liquid [1]. It is interesting that the shape of the streamer becomes filamentary by placing a solid such that the formation of gaseous cavities takes place exactly along the liquid-solid interface. The possible enhancement of the field between the front of the streamer head and the solid could be responsible to hold the propagation along the interface [11].

\section{CONCLUSIONS}

Charge and light intensity recordings as well as shadowgraphs of the propagation of streamers in mineral oil and along mineral oil-solid interfaces are recorded and analyzed. It is found that the recording of the charge injected by streamers changes with the solid material. The oil-solid interfaces with PVDF, PET and kraft fibril, paper with high permittivity and low surface roughness induce a quasicontinuous injection of charge during the early stage of the streamer propagation. The quasi-continuous injection of charge contributes to the increment of the average length and velocity of the streamer. Thus, the fastest and longest propagation of streamers is found with PVDF, PET and kraft fibril paper. This indicates that high permittivity and low surface roughness are strongly correlated with the increasing of the streamer length and velocity. The results obtained with the kraft paper show that high surface roughness is correlated with the short propagation of the streamer. The kraft paper case does not show a quasi-continuous injection of charge. Instead, it has a well-defined charge step in the early propagation of the streamer, similar to the case without solid. Furthermore, it is shown that the average streamer charge increases with the permittivity of the interface.

The results reported with PTFE and LDPE show that solids 
with similar permittivity to the mineral oil can affect the stopping voltage condition. The stopping voltage is approximately $14.7 \mathrm{kV}$ for PTFE, $13.3 \mathrm{kV}$ for LDPE and 12.2 $\mathrm{kV}$ with PVDF, PET, kraft fibril paper, kraft paper and without solid. The similar stopping voltage condition of the PVDF, PET films, the kraft fibril and kraft papers and the case without solid interface indicates that the stopping voltage condition is in these cases mainly depend on the processes taking place in the liquid phase.

\section{ACKNOWLEDGMENT}

The authors would like to acknowledge the financial support of ABB AB Corporate Research, the Swedish Centre for Smart Grids and Energy Storage SweGRIDS, the Swedish strategic research program StandUp for Energy and the EIT Innoenergy Materials platform to the research presented in the document. R. Hollertz also wants to acknowledge the financial support of the Swedish Energy Agency through the Elforsk program. L. Wågberg also acknowledges Walleenberg Wood Science Center for financial support.

\section{REFERENCES}

[1] O. Lesaint, "Prebreakdown phenomena in liquids: propagation 'modes' and basic physical properties," J. Phys. D. Appl. Phys., Vol. 49, No. 14, p. 144001, 2016.

[2] G. Massala and O. Lesaint, "Positive streamer propagation in large oil gaps: electrical properties of streamers," IEEE Trans. Dielectr. Electr. Insul., Vol. 5, No. 3, pp. 371-381, 1998.

[3] A. Saker and P. Atten, "Properties of streamers in transformer oil," IEEE Trans. Dielectr. Electr. Insul., Vol. 3, No. 6, pp. 784-791, 1996.

[4] P. Atten and A. Saker, "Streamer propagation over a liquid/solid interface," IEEE Trans. Electr. Insul., Vol. 28, No. 2, pp. 230-242, Apr. 1993.

[5] A. Beroual and L. Kebbabi, "Influence of the voltage waveform and hydrostatic pressure on morphology and final length of discharges propagating over solid-liquid interfaces," in IEEE Transactions on Dielectrics and Electrical Insulation, 2009, Vol. 16, No. 6, pp. 15741581 .

[6] L. Kebbabi and A. Beroual, "Optical and electrical investigations on creeping discharges over solid/liquid interfaces under impulse voltage," IEEE Int. Conf. Dielectr. Liq. 2005 ICDL 2005, pp. 127-130, 2005.

[7] H. Yamamoto, S. Uozaki, R. Hanaoka, S. Takata, Y. Kanamaru, and Y. Nakagami, "Creeping discharges in transformer oil under lightning impulse voltages over $100 \mathrm{kV}$ peak value," 2008 IEEE Int. Conf. Dielectr. Liq., pp. 1-4, 2008.

[8] R. Hanaoka, T. Kohrin, T. Miyagawa, and T. Nishi, "Creepage discharge characteristics over solid-liquid interfaces with grounded side electrode," IEEE Trans. Dielectr. Electr. Insul., Vol. 9, No. 2, pp. 308$315,2002$.

[9] J. Devins and S. Rzad, "Streamer Propagation in Liquids and over Liquid-Solid Interfaces," IEEE Trans. Electr. Insul., Vol. EI-17, No. 6, pp. 512-516, 1982.

[10] L. Lundgaard, D. Linhjell, G. Berg, and S. Sigmond, "Propagation of positive and negative streamers in oil with and without pressboard interfaces," IEEE Trans. Dielectr. Electr. Insul., Vol. 5, No. 3, pp. 388395, 1998.

[11] N. V. Dung, D. Linhjell, L. E. Lundgaard, H. K. Hoidalen, and M. Unge, "Effects of spatial restriction on streamers in white oil," in 2014 IEEE 18th Int'l. Conf. on Dielectric Liquids (ICDL), 2014, pp. 1-4.

[12] A. Beroual, V. H. Dang, M. L. Coulibaly, and C. Perrier, "Investigation on creeping discharges propagating over pressboard immersed in mineral and vegetable oils under AC, DC and lightning impulse voltages," IEEE Trans. Dielectr. Electr. Insul., Vol. 20, No. 5, pp. 1635-1640, 2013.

[13] Y. Nakao, M. Naruse, T. Sakai, H. Itoh, Y. Suzuki, Y. Sakai, and H. Tagashira, "Propagation characteristics of impulse creepage discharge in a parallel-plane gap with a protruding point in transformer oil," Electr. Eng. Japan, Vol. 124, No. 2, pp. 1-7, 1998.

[14] S. Ohgaki and Y. Tsunoda, "A Study of the Positive Streamer Growth under Surface Discharge Configuration in Liquid Paraffin," IEEE Trans. Electr. Insul., Vol. EI-19, No. 6, pp. 594-601, Dec. 1984.

[15] T. W. Liao and J. G. Anderson, "Propagation mechanism of impulse corona and breakdown in oil," Trans. Am. Inst. Electr. Eng. Part I Commun. Electron., Vol. 72, No. 5, pp. 641-648, 1953.

[16] B. Farazmand, "Study of electric breakdown of liquid dielectrics using Schlieren optical techniques," Br. J. Appl. Phys., Vol. 12, No. 5, pp. 251-254, 2002.

[17] E. O. Förster, "Progress in the Field of Electric Properties of Dielectric Liquids," IEEE Trans. Electr. Insul., Vol. 25, No. 1, pp. 45-53, 1990.

[18] Ø. L. Hestad, L. E. Lundgaard, and D. Linhjell, "New experimental system for the study of the effect of temperature and liquid to solid transition on streamers in dielectric liquids: Application to cyclohexane," IEEE Trans. Dielectr. Electr. Insul., Vol. 17, No. 3, pp. 764-774, 2010.

[19] S. Ingebrigtsen, L. E. Lundgaard, and P.-O. Åstrand, "Effects of additives on prebreakdown phenomena in liquid cyclohexane: II. Streamer propagation," J. Phys. D. Appl. Phys., Vol. 40, No. 18, pp. 5624-5634, 2007.

[20] O. Lesaint and T. V. Top, "Streamer initiation in mineral oil. Part I: Electrode surface effect under impulse voltage," IEEE Trans. Dielectr. Electr. Insul., Vol. 9, No. 1, pp. 84-91, 2002.

[21] A. Saker and P. Atten, "Potential distribution along single negative creeping streamer in transformer oil," IEE Proc. A Sci. Meas. Technol., Vol. 140, No. 5, p. 375, 1993.

[22] L. Lundgaard, D. Linhjell, G. Berg, and S. Sigmond, "Positive and negative streamers in oil gaps with and without pressboard interfaces," in ICDL'96. 12th Int'l. Conf. on Conduction and Breakdown in Dielectric Liquids, 1996, pp. 175-180.

[23] Q. Liu and Z. D. Wang, "Streamer characteristic and breakdown in synthetic and natural ester transformer liquids with pressboard interface under lightning impulse voltage," IEEE Trans. Dielectr. Electr. Insul., Vol. 18, No. 6, pp. 1908-1917, 2011.

[24] T. Mechlia, B. Gosse, A. Denat, and J. P. Gosse, "Electrophoretic Determination of the Charge at the Liquid-Solid Interface: Relation with Conduction Phenomena," IEEE Trans. Electr. Insul., Vol. EI-20, No. 2, pp. 365-370, Apr. 1985.

[25] H. Zainuddin, "Study of Surface Discharge Behaviour at the Oilpressboard Interface," Ph.D Thesis, University of Southampton, 2013.

[26] Q. Liu, Z. D. Wang, P. Dyer, and D. Walker, "Accumulative effect on streamer propagation of lightning impulses on oil/pressboard interface," in 2011 IEEE Int'l. Conf. on Dielectric Liquids, 2011, Vol. 5, No. 3, pp. $1-4$.

[27] A. Beroual and L. Kebbabi, "Influence of hydrostatic pressure on morphology and final length of creeping discharges over solid/liquid interfaces under impulse voltages," in 2008 IEEE Int'l. Conf. on Dielectric Liquids, ICDL 2008, 2008.

[28] M. G. Danikas, P. Atten, and A. Saker, "Streamer propagation over a liquid/solid interface," IEEE Trans. Dielectr. Electr. Insul., Vol. 1, No. 2, pp. 348-350, Apr. 1994.

[29] E. E. Kunhardt, L. G. Christophorou, and L. H. Luessen, The Liquid State and Its Electrical Properties, Vol. 193. Boston, MA: Springer US, 1988.

[30] L. Dumitrescu, O. Lesaint, N. Bonifaci, A. Denat, and P. Notingher, "Study of streamer inception in cyclohexane with a sensitive charge measurement technique under impulse voltage," J. Electrostat., Vol. 53, No. 2, pp. 135-146, Aug. 2001.

[31] A. Denat, J. P. Gosse, and B. Gosse, "Electrical conduction of purified 
cyclohexane in a divergent electric field," IEEE Trans. Electr. Insul., Vol. 23, No. 4, pp. 545-554, 1988.

[32] A. Beroual, C. Marteau, and R. Tobazeon, "Measurement of the charge of a single or of a series of streamers in liquids subjected to a voltage step.," IEEE Trans. Electr. Insul., Vol. 23, No. 3, pp. 427-431, 1988.

[33] C. T. Duy, O. Lesaint, a Denat, and N. Bonifaci, "Streamer propagation and breakdown in natural ester at high voltage," IEEE Trans. Dielectr. Electr. Insul., Vol. 16, No. 6, pp. 1582-1594, 2009.

[34] A. Beroual, M. Zahn, A. Badent, K. Kist, A. J. Schwabe, H. Yamashita, K. Yamazawa, M. Danikas, W. D. Chadband, and Y. Torshin, "Propagation and structure of streamers in liquid dielectrics," IEEE Electr. Insul. Mag., Vol. 14, No. 2, pp. 6-17, Mar. 1998.

[35] S. Ingebrigtsen, L. E. Lundgaard, and P.-O. Åstrand, "Effects of additives on prebreakdown phenomena in liquid cyclohexane: I. Streamer initiation," J. Phys. D. Appl. Phys., Vol. 40, No. 17, pp. 51615169, Sep. 2007.

[36] P. Gournay and O. Lesaint, "On the gaseous nature of positive filamentary streamers in hydrocarbon liquids. II: Propagation, growth and collapse of gaseous filaments in pentane," J. Phys. D. Appl. Phys., Vol. 27, No. 10, pp. 2117-2127, Oct. 1994

[37] R. Kattan, a Denat, and O. Lesaint, "Generation, growth, and collapse of vapor bubbles in hydrocarbon liquids under a high divergent electric field," J. Appl. Phys., Vol. 66, No. 9, pp. 4062-4066, 1989.

[38] Y. Tsunoda and S. Ohgaki, "A study of positive streamer growth under surface discharge configuration in liquid paraffin," No. 6, pp. 594-601, 1984.

[39] L. Dumitrescu, O. Lesaint, N. Bonifaci, A. Denat, and P. Notingher, "Study of streamer inception under impulse voltage in liquid cyclohexane," in 2000 Annual Report Conf. on Electrical Insulation and Dielectric Phenomena (Cat. No.00CH37132), 2000, Vol. 1, pp. 300-303.

[40] J. Nieto-Salazar, O. Lesaint, and A. Denat, "Transient current and light emission associated to the propagation of pre-breakdown phenomena in water," in 2003 Annual Report Conf. on Electrical Insulation and Dielectric Phenomena, 2003, pp. 542-545.

[41] L. Costeanu and O. Lesaint, "On mechanisms involved in the propagation of subsonic positive streamers in cyclohexane," Dielectr. Liq. 2002. ICDL 2002. Proc. 2002 IEEE 14th Int. Conf., No. Icdl, pp. 143-146, 2002.

[42] H. Yamashita, H. Kawai, K. L. Stricklett, and E. F. Kelley, "The effect of high pressure on prebreakdown phenomena in n-hexane," in 10th Int'l. Conf. on Conduction and Breakdown in Dielectric Liquids, 1990, Vol. 20899, pp. 404-409.

[43] M. Unge, S. Singha, N. Van Dung, D. Linhjell, S. Ingebrigtsen, and L. E. Lundgaard, "Enhancements in the lightning impulse breakdown characteristics of natural ester dielectric liquids," Appl. Phys. Lett., Vol. 102, No. 17, p. 172905, 2013.

[44] R. Hollertz, L. Wågberg, and C. Pitois, "Effect of composition and Morphology on the dielectric response of cellulose-based electrical insulation," IEEE Trans. Dielectr. Electr. Insul., Vol. 22, No. 4, pp. 2339-2348, 2015.

[45] H. Sehaqui, A. Liu, Q. Zhou, and L. A. Berglund, "Fast preparation procedure for large, flat cellulose and cellulose/inorganic nanopaper structures," Biomacromolecules, Vol. 11, No. 9, pp. 2195-2198, 2010.

[46] R. J. Taylor, "Effect of permittivity matching on the flashover of solid/liquid interfaces," Proc. Inst. Electr. Eng., Vol. 124, No. 10, p. $899,1977$.

[47] R. Kattan, A. Denat, and N. Bonifaci, "Formation of vapor bubbles in nonpolar liquids initiated by current pulses," IEEE Trans. Electr. Insul., Vol. 26, No. 4, pp. 656-662, 1991.

[48] O. Lesaint, P. Gournay, and R. Tobazeon, "Investigations on transient currents associated with streamer propagation in dielectric liquids," IEEE Trans. Electr. Insul., Vol. 26, No. 4, pp. 699-707, 1991.

[49] H. B. H. Sitorus, A. Beroual, R. Setiabudy, and S. Bismo, "Creeping discharges over pressboard immersed in jatropha curcas methyl ester and mineral oils," in 2015 IEEE 11th Int'l. Conf. on the Properties and Applications of Dielectric Materials (ICPADM), 2015, pp. 152155 .

[50] P. M. Mitchinson, P. L. Lewin, B. D. Strawbridge, and P. Jarman, "Tracking and surface discharge at the oil-pressboard interface," IEEE Electr. Insul. Mag., Vol. 26, No. 2, pp. 35-41, 2010.

[51] J.-W. G. Hwang, "Elucidating the mechanisms behind pre-breakdown phenomena in transformer oil systems," Thesis (Ph. D.)--Massachusetts Inst. Technol. Dept. Electr. Eng. Comput. Sci. 2010., 2010.

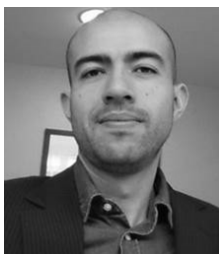

David Ariza received the B.Sc. and M.Sc. degrees from the National University of Colombia, Bogotá, in 2010 and 2012 respectively. He is currently doing his $\mathrm{Ph} . \mathrm{D}$. studies at the Royal Institute of Technology $\mathrm{KTH}$, at the Department of Electromagnetics of the School of Electrical Engineering, Stockholm. His main research interest is on the streamer propagation along dielectric liquid-solid interfaces.

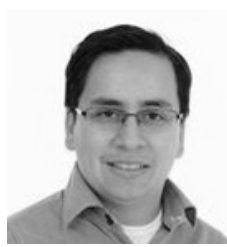

Marley Becerra (M'13) was born in Bogotá, Colombia. He received the B.Sc. and M.Sc. degrees in electrical engineering from the National University of Colombia, Bogotá, in 2000 and 2003, respectively, and the Ph.D. degree from the Division for Electricity and Lightning Research, Uppsala University, Uppsala, Sweden. He was involved in research with JENSEN Devices AB, Sollentuna, Sweden from 2004 to 2008. He joined ABB Corporate Research, Västerås, Sweden, in 2008, as a Scientist, where he is with the Electrical Apparatus and Diagnostics Group, Power Technologies. In 2010, he joined the School of Electrical Engineering, KTH Royal Institute of Technology, Stockholm, Sweden, as an Assistant Professor in smart grid power components. Since then, he has been with industry (ABB) and academia (KTH). His current research interests include lightning protection, lightning attachment processes, (thermal) plasmas in gases, liquids, and liquid/solid interfaces, multiphysics modeling, and applied physics for power system components. Dr. Becerra was a holder of a Guest Research Scholarship granted by the Swedish Institute, Uppsala, from 2003 to 2004.

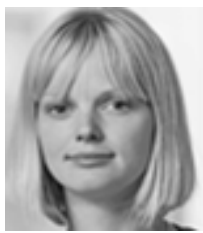

Rebecca Hollertz received the M.Sc. degree in Engineering Nano Science from Lund University, Sweden in 2008. She is currently pursuing her $\mathrm{PhD}$ studies at the Royal Institute of Technology (KTH), the Department of Fibre and Polymer Technology, Stockholm, Sweden. Her main research interest is in paper physics and chemistry and in particular in cellulose based insulation materials for electrical applications.

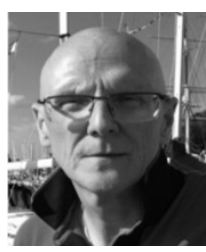

Lars Wågberg received a $\mathrm{PhD}$ in Paper Technology in the area of applied surface and colloid chemistry in 1987. After working for Innventia $\mathrm{AB}$, formerly STFI AB, he 1988 joined SCA Research AB in Sundsvall, Sweden, and worked for 8 years as a section manager doing applied research both in hygiene products and advanced paper products. Following this he was the research manager at SCA Research in Sundsvall until 2000 when he accepted a chair as professor in Technical Fibre Chemistry at MidSweden University in Sundsvall. After 2 years at MSU he was appointed a senior track chair as a professor in Fibre Technology at KTH, Stockholm, Sweden. Since then he has been doing research in applied physical chemistry as related to cellulose and cellulose based fibres and is today heading a research team of around 15 people.

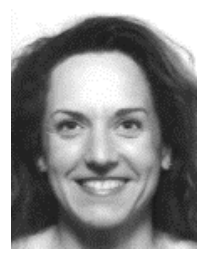

Claire Pitois received a $\mathrm{PhD}$ in Polymer Technology from the Royal Institute of Technology (KTH), Sweden. After performing a postdoc at UC Berkeley on solar cells, she joined the Rhodia Research Center in France to develop polymeric and inorganic solutions for industrial applications such as home care, personal care, oilfield and automotive. In 2008 she joined ABB AB Research Center in Sweden where she was successively project manager and principal scientist before to become manager of the Insulation and Material Technology group. Since 2015 she holds the position of Technology Manager for the product group distribution transformer. 